\section{Asociación del control glicémico con el apoyo familiar y el nivel de conocimientos en pacientes con diabetes tipo 2}

\author{
LAURA ÁVILA-JIMÉNEZ1,a, DOMINGO CERÓN O.², \\ ROSA ISELA RAMOS-HERNÁNDEZ ${ }^{3, \mathrm{~b}}$, LUBIA VELÁZQUEZ L. 3,c
}

\section{Association of family support and knowledge about the disease with glycemic control in diabetic patients}

Background: Family interventions may improve glycemic control among diabetic patients Aim: To evaluate the association of glycemic control with family support and level of knowledge in patients with type 2 diabetes. Material and Methods: Patients with type 2 diabetes completed a demographic survey. Glycosylated hemoglobin (HbAlc) was determined and glycemic control was defined as a value $<6,5 \%$. Two validated instruments were applied to evaluate family support, stratifying it in three categories (low, medium and high) and the level of knowledge about diabetes. Results: We studied 81 patients aged 32 to 65 years (53 females). Thirty six percent had an adequate glycemic control. Participants with a medium family support had three times greater risk of having an uncontrolled blood glucose than subjects with high family support. No significant association was found between the level of knowledge and glycemic control. Conclusions: Family support but not knowledge about the disease is associated with a better glycemic control of patients with type 2 diabetes.

(Rev Med Chile 2013; 141: 173-180).

Key words: Attitudes, practice; Blood glucose; Diabetes Mellitus, type 2; Family health.

\author{
'Delegación Morelos. Instituto \\ Mexicano del Seguro Social. \\ Cuernavaca Morelos. México. \\ ${ }^{2}$ Hospital Regional con Medicina \\ Familiar No.1. "Lic. Ignacio \\ García Téllez". Instituto Mexicano \\ del Seguro Social. Cuernavaca \\ Morelos. México. \\ ${ }^{3} U$ nidad de Investigación en \\ Epidemiología Clínica. Hospital \\ General Regional No 1 "Dr. Carlos \\ MacGregor Sánchez Navarro". \\ Instituto Mexicano del Seguro \\ Social. México D.F. \\ aDoctorado en Ciencias de la \\ Salud Pública en Epidemiología. \\ bMaestra en Ciencias Biológicas \\ con Mención en Nutrición. \\ 'Maestra en Ciencias en \\ Epidemiología Clínica. \\ Fuente de financiamiento: Este \\ estudio fue financiado por el \\ Instituto Mexicano del Seguro \\ Social "IMSS" a través del \\ otorgamiento de becas para \\ el programa de formación de \\ especialistas en Medicina Familiar \\ a uno de los coautores. \\ Recibido el 2 de marzo de 2011 , \\ aceptado el 4 de septiembre de \\ 2012. \\ Correspondencia a: \\ Laura Ávila. \\ Blvd. Benito Juárez Num. 18, Col. \\ Centro. Cuernavaca Morelos, \\ México. CP 62000. \\ Teléfono: (777) 3187632 \\ Fax: Oficina (777) 3189750 . \\ E-mail: laura.avilaj@gmail.com
}

L a diabetes mellitus tipo 2 (DM2) constituye un grave problema de salud pública. En Latinoamérica se ha documentado un incremento alarmante de este padecimiento con un descontrol metabólico. Sólo el 16,3\% de los adultos con DM2 con previo diagnóstico tienen control glicémico adecuado ${ }^{1}$. En México, la Encuesta Nacional de Salud y Nutrición "ENSANUT 2006" mostró una prevalencia de $7,3 \%$ de pacientes con DM2 con diagnóstico previo, y 7,1\% fue por hallazgo durante el estudio, 56,2\% presentó hemoglobina glicosilada $\left(\mathrm{HbA}_{\mathrm{lc}}\right)$ mayor de $11,1 \%{ }^{2}$.

Está documentado que la DM2 incrementa el riesgo de enfermedad coronaria, infarto, in- suficiencia renal, amputaciones y muerte, entre otras $^{3}$. Evidencias científicas muestran que las complicaciones por diabetes pueden ser prevenibles con un buen control metabólico, donde no sólo es importante la medición de la $\mathrm{HbA}_{1 c}$ sino también la educación del paciente con respecto a la enfermedad ${ }^{4,5}$. Los programas de educación en diabetes establecidos en las instituciones de salud tienen como objetivo educar al paciente ${ }^{6}$ ya que se ha demostrado que el conocimiento insuficiente con respecto a la enfermedad afecta negativamente el comportamiento y en consecuencia el autocuidado ${ }^{7}$.

Un manejo adecuado de la diabetes requiere 
cambios permanentes en el estilo de vida, para ello se requiere que el paciente y los miembros de la familia reciban educación para adquirir conocimientos y comprensión de la historia natural de la enfermedad; lo que permitirá que se desarrolle habilidades para un manejo adecuado de la diabetes a través del logro del control metabólico y prevención de complicaciones. Armour y cols. sugieren que la intervención de la familia es eficaz para mejorar el conocimiento y control glicémico de las personas con diabetes ${ }^{8}$ ya que el manejo efectivo de la enfermedad depende de la capacidad, habilidad del paciente y su red apoyo familiar para aprender y aplicar los conocimientos adquiridos ${ }^{9}$.

En la evaluación de conocimientos en pacientes con DM2, se han empleado varias metodologías para medir el control metabólico; a su vez se conocen diferentes intervenciones educativas ${ }^{10,11}$; combinando la educación pasiva y activa ${ }^{12,13}$, de forma individual ${ }^{14}$, o grupal ${ }^{15}$; así como el uso de instrumentos validados a nivel internacional ${ }^{16}$.

En México, algunos autores han implementado y validado instrumentos para evaluar el nivel de conocimientos en pacientes con diabetes ${ }^{17,18}$. Con respecto a la evaluación del apoyo familiar hay instrumentos validados: en el año 2003, Valadez y cols, propusieron un instrumento que mide el apoyo familiar en pacientes con DM2 ${ }^{19}$.

Dada la importancia de la educación en el paciente, así como el rol que juega el apoyo familiar sobre el manejo adecuado de la enfermedad, aunado a la limitada evidencia del uso de dos instrumentos con validez previa, que incluya la evaluación del apoyo familiar y nivel de conocimientos, el objetivo del presente estudio fue evaluar la asociación del control glicémico con el apoyo familiar y nivel de conocimientos en pacientes con DM2.

\section{Material y Métodos}

Se diseñó un estudio transversal analítico en pacientes con DM2 que acudieron al primer nivel de atención. Se realizó una entrevista en la consulta externa de Medicina Familiar en el Hospital General Regional con Medicina Familiar No 1 del Instituto Mexicano del Seguro Social (IMSS) en Cuernavaca, Morelos, México, de febrero a septiembre de 2008. El tamaño muestral estimado fue de 110 sujetos, se perdió $26 \%$ de la muestra, conformando una muestra final de 81 pacientes. Se calculó el poder estadístico empleando una fórmula para detectar como significativa una odds ratio de 3,08, basados en la distribución de frecuencias de apoyo familiar medio $(59,6 \%)$ y $(37,9 \%)$ encontrados en el estudio, un nivel de confianza de $95 \%$, y poder estadístico de $87,4 \%$.

Se incluyeron pacientes ambulatorios con DM2, con y sin hipertensión arterial sistémica (HAS), adscritos a alguno de los 19 consultorios de la Unidad de Medicina Familiar. La pesquisa se realizó a través del expediente clínico electrónico; identificando pacientes con diagnóstico DM2, se identificó la cita más próxima con su médico familiar y se les contactó. Se excluyeron a los pacientes con insuficiencia renal crónica, enfermedad vascular cerebral, insuficiencia respiratoria. Una vez corroborados los criterios de elegibilidad se solicitó la firma del consentimiento informado. Los sujetos elegibles fueron seleccionados a partir de un muestreo aleatorio utilizando las agendas electrónicas de cada consultorio, se identificaron a los pacientes con diagnóstico de DM2 con y sin HAS. Esta investigación fue aprobada por el Comité Local de Investigación del Hospital General Regional con Medicina Familiar No.1 del IMSS en Cuernavaca, Morelos. México, (Número de registro: R-2008-1701-11).

Los pacientes con descontrol glicémico fueron derivados al médico familiar y Servicio de Nutrición para ajustar tratamiento. Se excluyeron pacientes con insuficiencia renal, cáncer, demencia senil o depresión. Al inicio del estudio, se capacitó al equipo de investigación para la aplicación de los instrumentos de medición. El proceso de recolección de datos se inició en etapas en la primera etapa se aplicó una encuesta sobre características sociodemográficas y nivel de conocimientos de diabetes; definido como la habilidad o capacidad que tienen los sujetos para entender y manejar su enfermedad, sus causas y complicaciones. Se midió a través de un instrumento validado en población mexicana, el cual consta de 53 ítems con secciones sobre aspectos de la enfermedad, nutrición, ejercicio, monitoreo de glucosa, cuidados de pies y educación sobre diabetes. Cada sección aporta una puntuación y se clasifica de acuerdo a la metodología de Bautista y cols ${ }^{18}$ (Tabla 1).

En la segunda etapa, se programó para una cita al laboratorio clínico, previo ayuno de $8 \mathrm{~h}$, se tomó una muestra de $5 \mathrm{~mL}$ de sangre venosa para 
Control glicémico, apoyo familiar y conocimientos - L. Ávila-Jiménez et al

Tabla 1. Escala porcentual del nivel de conocimientos sobre diabetes ${ }^{1}$

\begin{tabular}{|clc|}
\hline Nivel de conocimientos & Puntos & Escala porcentual (\%) \\
\hline Bajo y/o nulo & 0 a 7 & 0 al 14 \\
\hline Básico & 8 a 26 & 15 al 50 \\
\hline Intermedio & 27 a 45 & 51 al 85 \\
\hline Avanzado & 46 a 53 & 86 al 100 \\
\hline
\end{tabular}

${ }^{1}$ Bautista-Martínez S, Aguilar-Salinas CA, Lerman I, Velasco ML, Castellanos R, Zenteno E, y cols. Diabetes Knowledge and Its Determinants in a Mexican Population. Diabetes Educ 1999; 25 (3): 374-81.

la determinación de la hemoglobina glicosilada $\left(\mathrm{HbA}_{1 \mathrm{c}}\right)$. Las muestras fueron colectadas en tubos vacutainer y procesadas en un equipo automatizado de alto rendimiento Sistemas Synchron Lx20 Pro Clinical System de Beckman Coulter ${ }^{\circledR}$, utilizando reactivos $\mathrm{HbA}_{1 \mathrm{c}}$ de los Sistemas Synchron con especificidad de $100 \%$ y sensibilidad de $95 \%$. Los resultados se integraron al expediente de cada paciente.

En la tercera etapa se aplicó el instrumento validado de apoyo familiar de 24 ítem, diseñado a través de un inventario de elementos para lograr el apoyo familiar construido a partir de apoyo emocional $<$ sentimientos de ser amado y de pertenencia, intimidad, el poder confiar en alguien, y disponibilidad de alguien con quien hablar $>$, apoyo tangible o instrumental <préstamo de ayuda directa o servicios $>$ y apoyo informacional $<$ brindar información, consejo, guía para resolver problemas > que consta de tres áreas: 1) Conocimientos de la familia relacionados con las medidas de control y complicaciones de la DM2 para brindar apoyo informacional; 2) Actitudes familiares hacia el enfermo como apoyo emocional y 3$)$ Medidas de control siendo el apoyo tangible. El apoyo familiar es clasificado en categorías de alto, medio y bajo de acuerdo con la puntuación obtenida. Para la calificación se utilizó la escala de Likert con cinco alternativas de respuestas (siempre, casi siempre, ocasionalmente, rara vez y nunca). La Tabla 2 muestra las categorías según el puntaje obtenido ${ }^{19}$. Se entregó el resultado de $\mathrm{HbA}_{1 \mathrm{c}}$ al paciente sugiriendo comunicarlo a su médico para los ajustes pertinentes. El control glicémico se evaluó con una sola medición de $\mathrm{HbA}_{1 c}$ y se consideró un control adecuado al valor $<6,5 \%{ }^{20}$. Al momento de aplicar los instrumentos los encuestadores desconocieron el resultado de hemoglobina glicosilada del paciente.

\section{Análisis estadístico}

Se realizó un análisis descriptivo de los datos, se calcularon medidas de tendencia central y dispersión, frecuencias y porcentajes. En el análisis bivariado se utilizó $\chi^{2}$ para las variables categóricas. Se calcularon las odds ratios para evaluar el conocimiento por categorías y de apoyo familiar. Se ajustó un modelo de regresión logística múltiple para evaluar el efecto conjunto del apoyo familiar y el nivel de conocimientos, controlando por tiempo de evolución de diabetes y comorbilidad como potenciales variables confundentes. Se estableció significación estadística con un valor de $\mathrm{p}<0,05$. El análisis estadístico se realizó en el software STATA/SE 9,0 para Windows.

\section{Resultados}

Durante el desarrollo de las etapas del estudio hubo 29 pérdidas; 14 faltaron a la cita de laboratorio clínico y 15 no acudieron a la cita para aplicación del instrumento de apoyo familiar, de los cuales 3 estaban hospitalizados. Las características demográficas y generales de la enfermedad, no fueron diferentes de aquellos sujetos incluidos en el estudio.

Tabla 2. Categorías para evaluar apoyo familiar en pacientes con $\mathrm{DM}^{2}$

\begin{tabular}{|c|c|}
\hline Apoyo familiar & Puntos \\
\hline Bajo & $51-119$ \\
\hline Medio & $120-187$ \\
\hline Alto & $188-265$ \\
\hline
\end{tabular}

${ }^{2}$ Valadez Figueroa I, Alfaro Alfaro N, Centeno Covarrubias G, Cabrera Pivaral C. Diseño de un instrumento para evaluar apoyo familiar al diabético tipo 2. Inv en Salud 2003; V (3): 167-72. 
Se incluyeron en el análisis a 81 personas con DM2. En la Tabla 3 se presentan las características sociodemográficas de la población estudiada. La edad fluctuó entre 32 y 65 años, con una mediana de edad de 53 años. Se observó predominio del sexo femenino $(65,4 \%)$. Con respecto al estado civil, más de $88 \%$ de los pacientes reportó estar casado o vivir en unión libre. El nivel de escolaridad reportado con mayor frecuencia fue primaria y secundaria completa $(70,4 \%)$ y preparatoria $17,3 \%$ respectivamente. Más de 50\% de las personas evaluadas fueron amas de casa y sólo un sujeto reportó no vivir con familiares.

La Tabla 4 describe las características generales de la enfermedad de los sujetos estudiados; se puede apreciar que 70,4\% de la población estudiada tuvo diagnóstico de DM2 entre los 31 a 50 años de edad, a su vez, $40,7 \%$ de los pacientes, presentaba menos de cinco años de ser diagnosticado y 29,6\% más de 10 años. El 45,7\% de los pacientes presentaba además, hipertensión arterial sistémica.

El tratamiento farmacológico reportado con mayor frecuencia $(76,5 \%)$ fue la utilización de antidiabéticos orales (ADO), como la glibenclamida, acarbosa, metformina y pioglitazona, disponibles

Tabla 3. Características sociodemográficas de los pacientes con DM2

\begin{tabular}{|c|c|c|}
\hline Características & $\mathbf{n}=\mathbf{8 1}$ & $\%$ \\
\hline Edad (años) & \multicolumn{2}{|c|}{$53(32-65)^{1}$} \\
\hline \multicolumn{3}{|l|}{ Sexo } \\
\hline Hombres & 28 & 34,6 \\
\hline Mujeres & 53 & 65,4 \\
\hline \multicolumn{3}{|l|}{ Estado civil } \\
\hline Casado o unión libre & 72 & 88,9 \\
\hline Soltero, divorciado, viudo & 9 & 11,1 \\
\hline \multicolumn{3}{|l|}{ Escolaridad } \\
\hline Primaria incompleta & 3 & 3,7 \\
\hline Primaria y secundaria completa & 57 & 70,4 \\
\hline Preparatoria o bachillerato & 14 & 17,3 \\
\hline Universidad & 7 & 8,6 \\
\hline \multicolumn{3}{|l|}{ Ocupación } \\
\hline Amas de casa & 43 & 53,1 \\
\hline No profesional ${ }^{2}$ & 35 & 43,2 \\
\hline Profesional & 3 & 3,7 \\
\hline Actualmente viven con familiares & 80 & 98,8 \\
\hline
\end{tabular}

'Mediana (Mínimo-Máximo). ²Incluyen: campesino, obrero, comerciante, empleado o empleada. en el Cuadro Básico Institucional de Medicamentos del IMSS(21). La mediana de la $\mathrm{HbA}_{1 \mathrm{c}}$ fue de $7,3 \%(3,7-13,9)$, se encontró un control glicémico adecuado $\left(\mathrm{HbA}_{1 \mathrm{c}}<6,5 \%\right)$ en $35,8 \%$ de los participantes.

En la Tabla 5 se muestra la distribución de frecuencias por control glicémico del paciente, y su relación con el apoyo familiar y el nivel de conocimientos. Se puede observar que $62 \%$ de los pacientes en control, contaban con apoyo familiar alto. En relación a los pacientes en descontrol glicémico el $60 \%$ tenía un apoyo familiar medio, y esto tuvo un valor de $\mathrm{p}=0,06$. En cuanto al nivel de conocimientos, no se encontró diferencia significativa entre las frecuencias observadas, en ambos grupos de pacientes controlados y descontrolados, la gran mayoría se ubicó en un nivel de conocimientos intermedio.

\section{Tabla 4. Características generales de la enfermedad de los pacientes con DM2 incluidos en el estudio}

\begin{tabular}{|c|c|c|}
\hline Características & $n=81$ & $\%$ \\
\hline \multicolumn{3}{|l|}{ Edad de diagnóstico de DM2 ${ }^{1}$} \\
\hline 18 a 30 años & 2 & 2,5 \\
\hline 31 a 50 años & 57 & 70,4 \\
\hline 51 a 65 años & 22 & 27,1 \\
\hline \multicolumn{3}{|l|}{ Años de diagnóstico de DM2 ${ }^{1}$} \\
\hline$<1$ año & 8 & 9,9 \\
\hline 1 a 5 años & 33 & 40,7 \\
\hline 6 a 10 años & 16 & 19,8 \\
\hline$>10$ años & 24 & 29,6 \\
\hline \multicolumn{3}{|l|}{ Tratamiento } \\
\hline $\mathrm{ADO}^{2}$ & 62 & 76,5 \\
\hline Insulina y/o ADO más dieta & 19 & 23,4 \\
\hline \multicolumn{3}{|l|}{$\begin{array}{l}\text { Condición de salud del paciente con } \\
\text { DM2 al momento de la entrevista }\end{array}$} \\
\hline $\mathrm{HAS}^{4}$ & 37 & 45,7 \\
\hline Dislipidemia $^{5}$ & 4 & 4,9 \\
\hline Ninguna & 33 & 40,7 \\
\hline Retinopatía & 1 & 1,2 \\
\hline Neuropatía & 6 & 7,4 \\
\hline $\mathrm{HbA}_{1 \mathrm{c}}(\%)^{6}$ & \multicolumn{2}{|c|}{$7,3(3,7-13,9)^{7}$} \\
\hline $\begin{array}{l}\text { Control glicémico }<6,5 \% \text { según } \\
\text { ALAD }^{8}\end{array}$ & 29 & 35,8 \\
\hline
\end{tabular}

'Diabetes mellitus tipo 2. ${ }^{2}$ Antidiabéticos Orales. ${ }^{3}$ Datos obtenidos del instrumento de conocimientos. ${ }^{4}$ Hipertensión Arterial Sistémica. ${ }^{5}$ Alteración de los niveles de colesterol y triglicéridos. ${ }^{6}$ Hemoglobina glucosilada. ${ }^{7}$ Mediana (Mínimo-Máximo). ${ }^{8}$ Asociación Latinoamericana de Diabetes (ALAD) 2007. 
Se calculó la odds ratio para evaluar la asociación entre el nivel de conocimientos y control glicémico, obteniéndose un valor de $\mathrm{OR}=0,34$ (IC 95\%: 0,8-1,30,) p = 0,06. Se identificó la necesidad de realizar un análisis estratificado para ajustar por variables.

Se ajustó un modelo de regresión logística multivariada en donde se identificó un cociente de máxima verosimilitud

Tabla 5. Análisis bivariado, distribución de frecuencias de apoyo familiar y nivel de conocimientos por control glicémico en pacientes con DM2

\begin{tabular}{|c|c|c|c|c|}
\hline & \multicolumn{2}{|c|}{$\begin{array}{c}\text { Control } \\
\text { glicémico }^{1} \\
<6,5 \% \mathrm{HbA1c}^{2} \\
(\mathrm{n}=29)\end{array}$} & \multicolumn{2}{|c|}{$\begin{array}{l}\text { Descontrol } \\
\text { glicémico } \\
\geq 6,5 \text { HbA1c } \\
(n=52)\end{array}$} \\
\hline & $\mathbf{n}$ & $\%$ & $\mathbf{n}$ & $\%$ \\
\hline \multicolumn{5}{|l|}{ Apoyo familiar* (puntos) } \\
\hline Alto de $188-265$ & 18 & 62,1 & 21 & 40,4 \\
\hline Medio de $120-187$ & 11 & 37,9 & 31 & 59,6 \\
\hline Bajo de 51 - 119 & 0 & -- & 0 & -- \\
\hline \multicolumn{5}{|c|}{ Nivel de conocimientos** (puntos) } \\
\hline Bajo y/o nulo & 0 & -- & 0 & -- \\
\hline Básico de 8 a 26 & 8 & 27,6 & 6 & 11,5 \\
\hline Intermedio de 27 a 45 & 20 & 68,9 & 43 & 82,7 \\
\hline Avanzado de 46 a 53 & 1 & 3,5 & 3 & 5,8 \\
\hline
\end{tabular}

${ }^{1}$ Fuente ALAD $2007{ }^{*} \chi^{2} p=0,061 ;{ }^{*} \chi^{2} p=0,180$.

Tabla 6. Modelo de regresión logística múltiple para la evaluación de la asociación entre el control glicémico y apoyo familiar ajustando por el nivel de conocimientos

\begin{tabular}{|c|c|c|c|}
\hline & OR & IC $95 \%$ & $\mathbf{P}$ \\
\hline \multicolumn{4}{|l|}{ Apoyo familiar (puntos) } \\
\hline Medio $120-187$ & 3,08 & $1,03-9,22$ & 0,043 \\
\hline Alto $188-265$ & $1^{*}$ & -- & -- \\
\hline \multicolumn{4}{|l|}{ Nivel de conocimientos (puntos) } \\
\hline Básico de 8 a 26 & 0,24 & $0,06-0,99$ & 0,050 \\
\hline Intermedio y avanzado de 27 a 53 & $1^{*}$ & -- & -- \\
\hline \multicolumn{4}{|l|}{ Años de diagnóstico de DM2 } \\
\hline$<1$ año & 1 * & -- & -- \\
\hline 2 a 5 años & 5,67 & $0,83-38,54$ & 0,076 \\
\hline 6 a 10 años & 2,31 & $0,30-17,70$ & 0,418 \\
\hline$>10$ años & 6,04 & $0,83-44,06$ & 0,076 \\
\hline \multicolumn{4}{|l|}{ Comorbilidad } \\
\hline Sin HAS 1 & 1 * & -- & -- \\
\hline Con HAS & 1,15 & $0,40-3,32$ & 0,790 \\
\hline
\end{tabular}

${ }^{*}$ Categoría de referencia. Log likelihood $=-45,842632$. Pseudo $R 2=0,1323$. Prob $>\chi^{2}=0,0298$. 'Hipertensión Arterial Sistémica. OR: Odds Ratio. de $-45,84$, la prueba de bondad de ajuste mostró un valor de $\mathrm{p}=0,029$; el hallazgo principal es que se encontró que los pacientes con DM2 que contaban con un apoyo familiar medio, tuvieron 3,08 veces la posibilidad de presentar un descontrol glicémico estimado a través de $\mathrm{HbA}_{1 c}(\geq 6,5 \%)$, con un valor de $\mathrm{p}=0,043$ comparados con un apoyo familiar alto. El modelo de regresión fue ajustado por nivel de conocimientos, años de diagnóstico y comorbilidades (Tabla 6).

\section{Discusión}

La DM2 es un problema de salud pública por su magnitud y repercusiones como causa de enfermedades cardiovasculares, renales, retinopatía y neuropatía ${ }^{22}$. Es importante que el paciente con diabetes participe activamente en el autocuidado, de tal manera que pueda evitar o retardar la presencia de las complicaciones propias de la misma ${ }^{23}$.

Los resultados del presente estudio muestran la asociación entre el apoyo familiar medio y el descontrol glicémico poniendo en relieve la importancia de una red familiar para el control de la enfermedad. Este hallazgo es similar a lo reportado por Méndez y cols., en 2004, donde mostraron una relación entre la disfunción familiar y mayor frecuencia de pacientes en descontrol glicémico de población ambulatoria del IMSS ${ }^{24}$. Asimismo, Alba y cols. en 2009, identificaron la importancia de una red de apoyo familiar para lograr el control metabólico integral de la enfermedad con mejora de la calidad de vida ${ }^{25}$.

El apoyo familiar vinculado a la alimentación ha sido demostrado por Epple y cols., en nativos Navajos con DM2, donde el apoyo familiar activo se asoció significativamente con el control de triglicéridos, colesterol y la disminución en los puntos porcentuales de la $\mathrm{HbA}_{1 \mathrm{c}}{ }^{26}$. Wen y cols., en 
2004 encontraron una asociación entre el apoyo familiar y el autocuidado, específicamente en la adherencia a la dieta y ejercicio en sujetos México americanos con $\mathrm{DM} 2^{27}$. De manera más reciente, Watanabe y cols., en 2010 identificaron al apoyo familiar como un componente crucial para el logro del control glicémico; su estudio mostró que los pacientes con DM2 con apoyo familiar en el área de nutrición mejoraron los niveles de $\mathrm{HbA}_{1 c}$ y triglicéridos ${ }^{28}$.

En la población estudiada, los resultados no muestran una asociación entre el nivel de conocimientos y el descontrol glicémico. Nuestros resultados concuerdan con lo publicado por GonzálezPedraza y cols., quienes no encontraron asociación entre el control metabólico de los pacientes y su nivel de conocimientos ${ }^{12}$. Esto es concordante con información previa que estableció que el nivel de conocimientos del paciente sobre la diabetes es bajo $^{12,29}$. No obstante, en un estudio más reciente se ha documentado que el control glicémico se logra cuando el nivel de conocimientos de los pacientes sobre diabetes es alto ${ }^{30}$.

El nivel de conocimientos sobre la enfermedad mejora a través de la aplicación de diferentes estrategias de educación dirigidas al paciente, al inicio y durante la evolución de la enfermedad, las cuales deben ser abordadas desde el primer nivel de atención ${ }^{13,31}$. Esto se ha demostrado cuando estrategias educativas a nivel individual o grupal evidencian un impacto positivo en el control de la enfermedad ${ }^{11,32}$. situación que no se observa al brindar atención médica sin incluir estrategias educativas $^{33,34}$.

El nivel de conocimientos que poseen nuestros pacientes, sobre la diabetes no tuvo una gran variabilidad y no permitió identificar la asociación esperada. De igual forma la proporción del control glicémico fue mayor a lo reportado por la Encuesta Nacional de Salud y Nutrición desarrollada en México en el 2006 35 ; esto se explica, en parte, porque en el IMSS a partir del año 2002 se implantaron los Programas Integrados de Salud (PREVENIMSS) que es una estrategia de prestación de servicios que tiene como propósito general la provisión sistemática y ordenada de acciones relacionadas con la promoción y educación para la salud ${ }^{36}$.

Los resultados de este estudio tienen implicaciones para seguir fortaleciendo los esfuerzos sobre los procesos educativos centrados en el paciente.
El equipo de salud debe incorporar la evaluación de la red de apoyo familiar con que cuenta el paciente con diabetes y el conocimiento de sus condiciones específicas, independientemente de sus conocimientos sobre la enfermedad. Debe ser una prioridad durante el proceso de atención médica la optimización de las oportunidades asistenciales para aumentar el nivel de autocuidado.

En conclusión, nuestros resultados confirman la importancia del apoyo familiar en el control glicémico del paciente con DM2 que favorezca la modificación y permanencia de estilos de vida saludables.

Agradecimientos: Los autores agradecen a los pacientes participantes y a los médicos de cada uno de los consultorios de Medicina Familiar, además al Laboratorio Clínico, en particular a la Dra. Marina Pilar Gómez Narváez por su gestión y apoyo.

\section{Referencias}

1. Escobedo J, Buitrón LV, Velasco MF, Ramírez JC, Hernández R, Macchia A, et al. High prevalence of diabetes and impaired fasting glucose in urban Latin America: the CARMELA Study. Diabetic Medicine 2009; 26 (9): 864.

2. Villalpando S, de la Cruz V, Rojas R, Shamah-Levy T, Ávila M, Gaona B, et al. Prevalence and distribution of type 2 diabetes mellitus in Mexican adult population: a probabilistic survey. Salud Pública Mex 2010; 52 (Suppl 1): S19-26.

3. Stratton I, Adler A, Neil H, Matthews D, Manley S, Cull $\mathrm{C}$, et al. Association of glycaemia with macrovascular and microvascular complications of type 2 diabetes (UKPDS 35): prospective observational study. BMJ 2000; 321 (7258): 405-12.

4. Deakin T, McShane C, Cade J, Williams R. Group based training for self-management strategies in people with type 2 diabetes mellitus. Cochrane Database Syst Rev 2005; 18 (2): CD003417.

5. Álvarez Guisasola F, Mavros P, Nocea G, Alemao E, Alexander C, Yin D. Glycaemic control among patients with type 2 diabetes mellitus in seven European countries: findings from the Real-Life Effectiveness and Care Patterns of Diabetes Management (RECAP-DM) study. Diabetes Obes Metab 2008; (Suppl 1): 8-15.

6. IMSS, Dirección de Prestaciones Médicas, Unidad de Atención Médica, Coordinación de Áreas Médicas. Programa Institucional de Prevención y Atención al Paciente Diabético. División de Medicina Familiar, editor. 
México, DF: IMSS; septiembre 2008.

7. Ceballos RM, Coronado GD, Thompson B. Having a Diagnosis of Diabetes Is Not Associated With General Diabetes Knowledge in Rural Hispanics. J Rural Health 26 (4): 342.

8. Armour T, Norris S, Jack LJ, Zhang X, Fisher L. The effectiveness of family interventions in people with diabetes mellitus: a systematic review. Diabet Med 2005; 22 (10): 1295-305.

9. White P, Smith SM, Hevey D, O'Dowd T. Understanding Type 2 Diabetes. Diabetes Educ 2009; 35 (5): 810-7.

10. Mollaoğlu M, Beyazit E. Influence of diabetic education on patient metabolic control. Appl Nurs Res 2009; 22 (3): 183-90.

11. Flores-López M, Velázquez-Tlapanco J, CamachoCalderón N. Effectiveness of a strategy in type 2 diabetic patient education in relation to metabolic control, nutrition and blood pressure. Rev Med Inst Mex Seguro Soc 2008; 46 (3): 301-10.

12. González-Pedraza Avilés A, Alvara-Solís EP, MartínezVázquez R, Ponce-Rosasa RE. [Disease knowledge among type 2 diabetes mellitus patients attending primary care]. Gac Med Mex 2007; 143 (6): 453-62.

13. Herenda S, Tahirovic H, Poljakovic D. Impact of education on disease knowledge and glycaemic control among type 2 diabetic patients in family practice. Bosn J Basic Med Sci 2007; 7 (3): 261-5.

14. Duke Sally-Anne S, Colagiuri S, Colagiuri R. Individual patient education for people with type 2 diabetes mellitus. Cochrane Database of Systematic Reviews. 2009 (1).

15. Lara-Esqueda A, Aroch-Calderón A, Jiménez R, ArceoGuzmán M, Velázquez-Monroy O. Grupos de Ayuda Mutua: Estrategia para el control de diabetes e hipertensión arterial. Arch Cardiol Mex 2004; 74 (4): 330-6.

16. Hess GE, Davis WK. The Validation of a Diabetes Patient Knowledge Test. Diabetes Care 1983; 6 (6): 591-6.

17. Campo J, Vargas M, Martínez-Terrer T, Cía P. Adaptación y validación de conocimientos sobre la diabetes mellitus. Aten Primaria 1992; 9: 100-5.

18. Bautista-Martínez S, Aguilar-Salinas CA, Lerman I, Velasco ML, Castellanos R, Zenteno E, et al. Diabetes Knowledge and Its Determinants in a Mexican Population. Diabetes Educ 1999; 25 (3): 374-81.

19. Valadez Figueroa I, Alfaro Alfaro N, Guillermo CC, Cabrera Pivaral C. Diseño de un Intrumento para evaluar apoyo familiar al diabético tipo 2. Inv en salud 2003; V (3): 167-72.

20. Asociación Latinoamericana de Diabetes. Capítulo 4. Control clínico y metabólico de la DM2 (Actualización 2006). In: Asociación Latinoamericana de Diabetes, editor. Guias ALAD 2000 para el diagnóstico y manejo de la Diabetes Mellitus Tipo 2 con medicina basada en evidencia: ALAD; 2006. p. 16-9.

21. IMSS. Cuadro Básico Institucional de Medicamentos. México, DF: IMSS; 2009 [cited 2011]; enero]. Available from: http://www.imss.gob.mx/transparencia/cuadros/ medicamentos.htm.

22. Intensive blood-glucose control with sulphonylureas or insulin compared with conventional treatment and risk of complications in patients with type 2 diabetes (UKPDS 33). Lancet 1998; 352 (9131): 837.

23. Augstein P, Vogt L, Kohnert K, Heinke P, Salzsieder E. Translation of personalized decision support into routine diabetes care. J Diabetes Sci Technol 2010; 4 (6): 1532-9.

24. Méndez-López D, Gómez-López V, García-Ruiz M, Pérez-López J, Navarrete- Escobar H. Disfunción familiar y control del paciente diabético tipo 2. Rev Med IMSS 2004; 42 (4): 281-4.

25. Alba L, Bastidas C, Vivas J, Gil F. Prevalence of glycemic control and associated factors in type 2 diabetes mellitus patients at the Hospital Universitario de San Ignacio, Bogotá-Colombia. Gac Med Mex 2009; 145 (6): 469-74.

26. Epple C, Wright AL, Joish VN, Bauer M. The Role of Active Family Nutritional Support in Navajos' Type 2 Diabetes Metabolic Control. Diabetes Care 2003; 26 (10): 2829-34.

27. Wen LK, Shepherd MD, Parchman ML. Family Support, Diet, and Exercise Among Older Mexican Americans With Type 2 Diabetes. Diabetes Educ 2004; 30 (6): 980 93.

28. Watanabe K, Kurose T, Kitatani N, Yabe D, Hishizawa $\mathrm{M}$, Hyo T, et al. The role of family nutritional support in Japanese patients with type 2 diabetes mellitus. Intern Med 2010; 49 (11): 983-9.

29. Çaliskan D, Ozdemir O, Ocaktan E, Idil A. Evaluation of awareness of Diabetes Mellitus and associated factors in four health center areas. Patient education and counseling 2006; 62 (1): 142-7.

30. Ozcelik F, Yiginer O, Arslan E, Serdar M, Uz O, Kardesoglu E, et al. Association between glycemic control and the level of knowledge and disease awareness in type 2 diabetic patients. Pol Arch Med Wewn 2010; 120 (10): 399-406.

31. Chlebowy DO, Garvin BJ. Social Support, Self-efficacy, and Outcome Expectations. Diabetes Educ 2006; 32 (5): 777-86.

32. Cabrera-Pivaral C, González-Pérez G, Vega-López M, Arias-Merino E. Impact of participatory education on body mass index and blood glucose in obese type-2 diabetics. Rev Med Inst Mex Seguro Soc 2008; 46 (3): 301-10. 
33. Labhardt N, Balo J-R, Ndam M, Grimm J-J, Manga E. Task shifting to non-physician clinicians for integrated management of hypertension and diabetes in rural Cameroon: a programme assessment at two years. BMC Health Services Research 2010; 10: 339.

34. Sönnichsen A, Winkler H, Flamm M, Panisch S, Kowatsch P, Klima G, et al. The effectiveness of the Austrian disease management programme for type 2 diabetes: a cluster-randomised controlled trial. BMC Fam Pract 2010; 11: 86.
35. Olaiz-Fernández G, Rivera-Dommarco J, ShamahLevy T, Rojas R, Villalpando-Hernández S, Hernández-Ávila M, et al. Encuesta Nacional de Salud y Nutrición 2006. Cuernavaca: Instituto Nacional de Salud Pública; 2006.

36. Gutiérrez-Trujillo G, Flores-Huerta S, Fernández-Gárate IH, Martínez-Montañez OG, Velasco-Murillo V, Fernández-Cantón S, et al. Estrategia de prestación y evaluación de servicios preventivos. Rev Med Inst Mex Seguro Soc 2006; 44 (Supl 1): S3-S21. 